\title{
METLAPILCOATLUS NUMMIFER (VIPERIDAE)
}

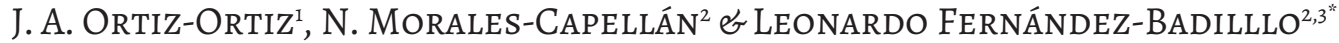

${ }^{1}$ I1Colegio de estudios científicos y tecnológicos del Estado de Hidalgo, Plantel Coacuilco, Huejutla de Reyes, Hidalgo, 43010, México.

${ }^{2}$ Predio Intensivo de Manejo de Vida Silvestre X-Plora Reptilia, km 65 carretera México-Tampico, Pilas y Granadas, Metztitlán, Hidalgo, 43350, México. ${ }^{3}$ Centro de Investigaciones Biológicas, Universidad Autónoma del Estado de Hidalgo. Km 4.5 carretera Pachuca-Tulancingo, s/n, Mineral de la Reforma, Hidalgo, 42184, México.

Correspondence:fernandezbadillo8o@gmail.com

Received: 2021-02-10. Accepted: 2021-04-28.

México: Hidalgo: Municipio de Huautla, localidad $16 \mathrm{~km}$ al suroeste de la localidad de Hernandeztla $\left(21.008323^{\circ} \mathrm{N}, 98.318347^{\circ} \mathrm{O}\right.$, WGS 84; 506 m s.n.m.), 2 de agosto de 2020 a las 09:00 h. Un individuo fue fotografiado por Eva Pineda Bravo (MZFZ-IMG 301; Fig. 1), en un lugar conocido por los habitantes como "Cueva del Tigre". Este avistamiento representa el primer registro de Metlapilcoatlus nummifer para el municipio de Huautla, y amplía la distribución de la especie en $39.6 \mathrm{~km}$ al este de la localidad más cercana, en Quetzaltzongo, Tlanchinol, Hidalgo (RamírezBautista et al., 2010). M. nummifer también ha sido registrada para otros municipios del estado como Huehuetla, Molango, Jacala de Ledezma, Pisaflores, Tlanchinol y Tepehuacán de Guerrero (Ramírez-Bautista et al., 2010; 2014; Raño-Hernández, 2016; Fernández-Badillo et al., 2017). El ejemplar fue observado arriba de un árbol a una altura de aproximadamente dos metros, lo cual resulta interesante, ya que comúnmente se menciona que es una especie de hábitos terrestres (Campbell \& Lamar, 2004; Heimes, 2016).

Agradecimientos.- A Uri Omar García-Vázquez, por permitirnos depositar la fotografía en la colección herpetológica MZFZ de la UNAM.

\section{LITERATURA CITADA}

Campbell, J.A., \& W.W. Lamar. 2004. The venomous reptiles of the Western Hemisphere, vol. 1. Comstock, Ithaca, New York.

Fernández-Badillo, L., N. Morales-Capellán, C. R. Olvera-Olvera, G. Montiel-Canales \& I. Goyenechea. Guía de las serpientes del estado de Hidalgo. Universidad Autónoma del Estado de Hidalgo. Pachuca, Hidalgo, México.

Ramírez-Bautista, A., U. Hernández-Salinas, F. Mendoza-Quijano, R. Cruz-Elizalde, B.P Stephenson, V.D. Vite-Silva \& A. LeyteManrique. 2010. Lista anotada de los anfibios y reptiles del estado de Hidalgo, México. Universidad Autónoma del Estado de Hidalgo/Comisión Nacional para el Conocimiento y Uso de la Biodiversidad. Pachuca, Hidalgo, México.
Ramírez-Bautista, A., U. Hernández-Salinas, R. Cruz-Elizalde, C. Berriozabal-Islas, D. Lara-Tufiño, I. Goyenechea \& J. M. Castillo-Cerón. 2014. Los anfibios y reptiles de Hidalgo, México: Diversidad, Biogeografía y Conservación. Sociedad Herpetológica Mexicana. Pachuca, Hidalgo, México.

Heimes, P. 2016. Herpetofauna Mexicana Vol.1. Snakes of Mexico. Edition Chimaira. Frankfurt am Main, Alemania.

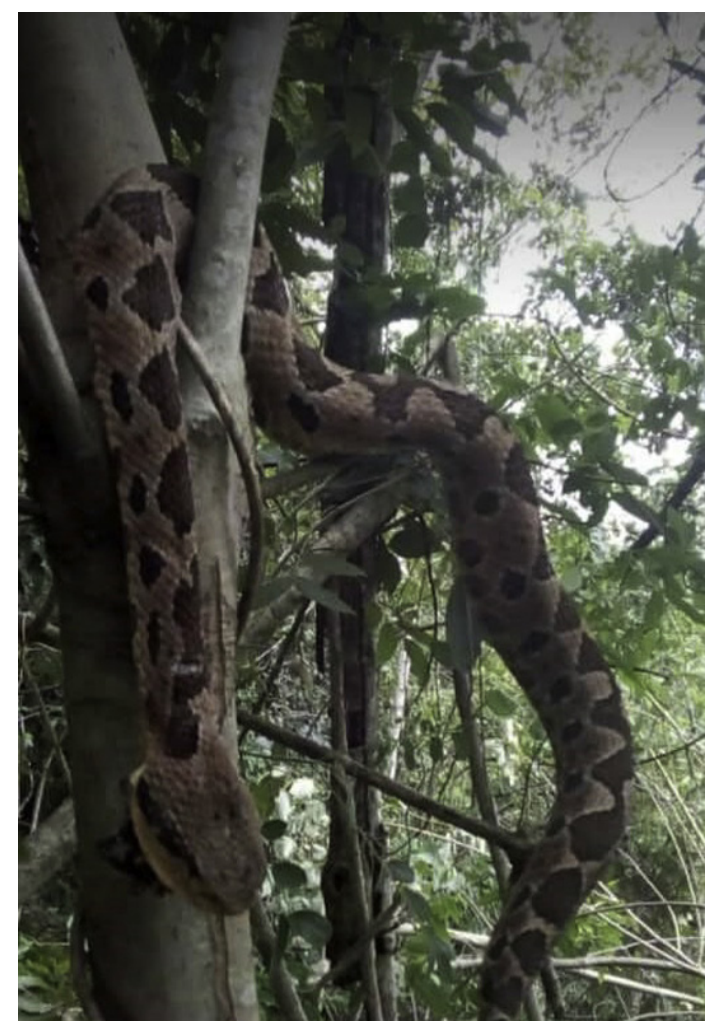

Figure 1. Metlapilcoatus nummifer from Hernaneztla, municipality of Huautla, Hidalgo, Mexico (MZFZ-IMG 301). Photo: Eva Pineda-Bravo.

Figura 1. Metlapilcoatus nummifer Hernandeztla, municipio de Huautla, Hidalgo, México (MZFZ-IMG 301). Foto: Eva Pineda-Bravo. 